\title{
CORRIGENDUM
}

\section{Should community-based services be publicly funded or contracted out? - CORRIGENDUM}

\author{
Vancouver Coastal Health Community, Vancouver, British Columbia, Canada \\ doi: 10.1017/S1478951512000454, Published by Cambridge University Press, 6 July 2012
}

The above degree of MPH will not be awarded until 2013. The degree citation should read: MPH(Candidate) -Lynnette Nathalie Lyzwinski, BA, MPH 